Journal of Applied AnALysis

Vol. 6, No. 2 (2000), pp. 213-226

\title{
GRAPH CONVERGENCE OF SET-VALUED MAPS AND ITS RELATIONSHIP TO OTHER CONVERGENCES
}

\author{
I. DEL PRETE, M. DI IORIO AND L̆. HOLÁ
}

Received June 1, 1999 and, in revised form, February 28, 2000

\begin{abstract}
The notion of even-outer-semicontinuity for set-valued maps is introduced and compared with related ones from [4] and [11]. The coincidence of these notions provides a new characterization of compactness and of local compactness. The following result is proved: Let $X$ be a topological space, $Y$ a uniform space, $\left\{F_{\sigma}: \sigma \in \Sigma\right\}$ be a net of set-valued maps from $X$ to $Y$ and $F$ be a set valued map from $X$ to $Y$. Then any two of the following conditions imply the third: (1) the net $\left\{F_{\sigma}: \sigma \in \Sigma\right\}$ is evenly-outer semicontinuous; (2) the net $\left\{F_{\sigma}: \sigma \in \Sigma\right\}$ is graph convergent to $F$; (3) the net $\left\{F_{\sigma}: \sigma \in \Sigma\right\}$ is pointwise convergent to $F$. This theorem generalizes some results from [4] and [11].
\end{abstract}

Graph convergence (that is Painlevé-Kuratowski convergence of graphs) of set-valued maps was studied in many books and papers (see for example $[1,2,4,9,11])$. In this topic we can include also graph convergence of single-valued maps [5, 12, 13], epiconvergence of lower semicontinuous functions $[4,6,7]$ as well as Painlevé-Kuratowski convergence of graphs of partial maps [8]. In the books of Attouch [1], Aubin-Frankowska [2]

1991 Mathematics Subject Classification. 54C60, 54B20.

Key words and phrases. Set-valued map, graph convergence, pointwise convergence, continuous convergence, even-outer-semicontinuity.

Work partially supported by CNR and MURST.

The third author is grateful to the first two for their kind hospitality during her stay as visiting professor at the University of Naples.

ISSN 1425-6908 C Heldermann Verlag. 
and Rockafellar-Wets [14] can be found many applications of this convergence to variational and optimization problems, differential equations and approximation theory. Graph convergence of preference relations is used also in mathematical economics [3]. However graph convergence of maps, even when they are single valued, is not always easy to verify. In our paper we study the relationship between graph convergence and pointwise one introducing the notion of even-outer-semicontinuity which allows us to pass from one convergence to the other. Other notions of joint continuity (see for example $[4,9,11]$ ) are compared with ours. The relationship between the notions of continuous convergence and graph convergence is analysed too.

\section{Preliminaries}

If $Y$ is a topological space and $y \in Y, U_{y}$ ( or $U$ if there is no possibility of confusion) will denote a general nbd (neighborhood) of $y$ and $\mathcal{U}(y)$ the set of all open nbds of $y . K(Y)$ and $2^{Y}$ will denote the sets of all compact and all closed subsets of $Y$ respectively.

Let us recall [5] that a net $\left\{A_{\sigma}: \sigma \in \Sigma\right\}$ of subsets of $Y$ is said to be:

- $K^{+}$-convergent to a subset $A$ of $Y$ if Ls $A_{\sigma} \subset A$, where Ls $A_{\sigma}=$ $\left\{y \in Y\right.$ : each nbd of $y$ intersect $A_{\sigma}$ for all $\sigma$ in some cofinal subset of $\Sigma\}$;

- $K^{-}$-convergent to $A$ if $A \subset \operatorname{Li} A_{\sigma}$, where Li $A_{\sigma}=\{y \in Y$ : each nbd of $y$ intersect $A_{\sigma}$ in some residual subset of $\Sigma$; ;

- topologically convergent (or Painlevé-Kuratowski convergent) to $A$, (and we will write Lt $A_{\sigma}=A$ ) if $\operatorname{Li} A_{\sigma}=\operatorname{Ls} A_{\sigma}=A$.

If $Y$ is completely regular, $\gamma$ is a compatible uniformity on $Y$ and $A$ is closed, it is easy to prove that:

- Ls $A_{\sigma} \subset A$ if and only if for every $y \in Y$ and every $V$ in $\gamma$ there is a $\operatorname{nbd} U_{y}$ of $y$ and $\sigma_{0} \in \Sigma$ such that for every $\sigma \geq \sigma_{0}$

$$
A_{\sigma} \cap U_{y} \subset V[A]
$$

- $A \subset \operatorname{Li} A_{\sigma}$ if and only if for every $y \in Y$ and every $V$ in $\gamma$ there is a $\operatorname{nbd} U_{y}$ of $y$ and $\sigma_{0} \in \Sigma$ such that for every $\sigma \geq \sigma_{0}$

$$
A \cap U_{y} \subset V\left[A_{\sigma}\right] .
$$

If $X$ is a topological space, $C\left(X, 2^{Y}\right)$ will be the family of all continuous set-valued maps from $X$ to $Y$ with closed values and, if $F$ is a set-valued map from $X$ to $Y$, Gr $F$ will denote the graph of $F$, that is

$$
\text { Gr } F=\{(x, y) \in X \times Y: y \in F(x)\} \text {. }
$$

If $F$ and $F_{\sigma}$ are set-valued maps from $X$ to $Y$, we will say that: 
- the net $\left\{F_{\sigma}: \sigma \in \Sigma\right\}$ is pointwise convergent at $x \in X$ to $F$ if $F(x)=$ Lt $F_{\sigma}(x)$; is pointwise convergent in $X$ if this holds for every $x \in X$,

- the net $\left\{F_{\sigma}: \sigma \in \Sigma\right\}$ is graph-convergent to $F$ if Gr $F=\operatorname{Lt} \operatorname{Gr} F_{\sigma}$.

For any undefined terms see $[6,10]$.

It can be proved that if $X$ is a discrete topological space, graph convergence coincides with pointwise one (see [11] for sequences of set-valued maps), while in general neither graph nor pointwise convergence implies the other. We can even characterize discreteness by this coincidence, as the following theorems show.

First we recall that $C\left([0,1], 2^{Y}\right)$ is non-trivial if there exists a continuous set-valued function satisfying the condition $F(0) \neq F(1)$.

The following example shows that there is such a topological space $Y$ for which $C\left([0,1], 2^{Y}\right)$ is non-trivial but $C([0,1], Y)$ is trivial.

Example 1.1. If $X=[0,1]$ is equipped with the natural topology, $Y=$ $\{0,1,2\}$ is equipped with the topology $\mathcal{T}$, having as open sets $\{0\}$ and $\{1,2\}$, then $C([0,1], Y)$ is trivial while $C\left([0,1], 2^{Y}\right)$ is non-trivial, since $F$ defined by

$$
F(x)=\left\{\begin{array}{lll}
\{0,1\} & \text { if } & x \in[0,1) \\
\{0,1,2\} & \text { if } \quad x=1
\end{array}\right.
$$

is continuous and non trivial.

Now we can prove:

Proposition 1.2. Let $X$ be a Tychonoff non discrete space and $Y$ be such that $C\left([0,1], 2^{Y}\right)$ is not trivial. There is a net $\left\{F_{\sigma}: \sigma \in \Sigma\right\}$ in $C\left(X, 2^{Y}\right)$ pointwise convergent to $F \in C\left(X, 2^{Y}\right)$ which fails to be graph convergent to $F$.

Proof. Let $x_{0}$ be a non-isolated point in $X$ and for every open nbd $U$ of $x_{0}$ choose a point $x_{U} \in U \backslash\left\{x_{0}\right\}$. There is a continuous function $h_{U}$ from $X$ to $[0,1]$ satisfying the conditions $h_{U}\left(x_{U}\right)=1$ and $h_{U}(x)=0$ for every $x \in(X \backslash U) \cup\left\{x_{0}\right\}$. There is a continuous set-valued map $H$ from [0,1] to $2^{Y}$ such that $H(1) \backslash H(0) \neq \emptyset$. For every open nbd $U$ of $x_{0}$ the set-valued map $F_{U}=H \circ h_{U}$ is continuous and the net $\left\{F_{U}: U \in \mathcal{U}\left(x_{0}\right)\right\}$ is pointwise convergent to $F$ where $F(x)=H(0)$ at every $x$. Indeed if $x \neq x_{0}$ and $U$ is an open nbd of $x_{0}$ such that $x \notin U$, for every nbd $U^{\prime} \geq U$ it results $F_{U^{\prime}}(x)=H(0)$. If $x=x_{0}$, then $F_{U}\left(x_{0}\right)=H(0)$ for every nbd $U$. However Lt $\operatorname{Gr} F_{U} \neq \operatorname{Gr} F$, since $\left(x_{0}, y\right) \in \operatorname{Li}$ Gr $F_{U}$ if $y \in H(1) \backslash H(0)$. 
Proposition 1.3. Let $X$ be a Tychonoff non discrete space and $Y$ be a normal space such that $C\left([0,1], 2^{Y}\right)$ is not trivial. There is a net $\left\{F_{\sigma}\right.$ : $\sigma \in \Sigma\}$ in $C\left(X, 2^{Y}\right)$ graph convergent to $F \in C\left(X, 2^{Y}\right)$ which fails to be pointwise convergent to $F$.

Proof. There is $h \in C\left([0,1], 2^{Y}\right)$ such that $h(1) \backslash h(0) \neq \emptyset$. It is easy to prove that the set-valued map, from $[0,1]$ to $Y, H(x)=\overline{\bigcup_{s \leq x} h(s)}$ is continuous. Let $x_{0}$ be a non-isolated point in $X$. For every open nbd $U$ of $x_{0}$, let $f_{U}$ be a continuous function from $X$ to $[0,1]$ such that $f_{U}\left(x_{0}\right)=0$ and $f_{U}(X \backslash U)=1$. If we put $F_{U}=H \circ f_{U},\left\{F_{U}: U \in \mathcal{U}\left(x_{0}\right)\right\}$ is a net of continuous set-valued maps which graph converges to $F$, where $F(x)=H(1)$ for every $x \in X$, but does not pointwise converges to $F$ since Lt $F_{U}\left(x_{0}\right)=h(0)$.

\section{Continuity and equicontinuity}

In this part, for nets of set-valued maps, we introduce the notion of evenouter-semicontinuity and even-inner-semicontinuity and compare them with others known in the literature.

It is well known that for closed-valued maps between topological spaces there are various notions of continuity connected with the convergence or topology supported by $2^{Y}$. If the values belong to a uniform space $(Y, \gamma)$, when $2^{Y}$ is equipped with $K^{+}$or $K^{-}$convergence we obtain the following definitions:

Remark 2.1. A set-valued map $F$ from $X$ to $Y$, closed-valued in $x$, is outer-semicontinuous at $x$ if for every $V$ in $\gamma$ and for every $y \in Y$ there is a nbd $U_{x}$ of $x$, a nbd $U_{y}$ of $y$ such that, for every $z \in U_{x}$ it results:

$$
F(z) \cap U_{y} \subset V[F(x)] .
$$

Remark 2.2. A set-valued map $F$ from $X$ to $Y$, closed-valued in $x$, is lower or inner-semicontinuous at $x$ if for every $V$ in $\gamma$ and for every $y \in Y$ there is a nbd $U_{x}$ of $x$, a nbd $U_{y}$ of $Y$ such that, for every $z \in U_{x}$ it results

$$
F(x) \cap U_{y} \subset V[F(z)] .
$$

In the literature there are many definitions of joint continuity for singlevalued or set-valued maps $[9,10,14]$. For nets of set-valued maps with values in a uniform space we give the following definitions which absorb the classical definitions of equicontinuity and even-continuity for a family of functions due to Kelley [10]. 
Definition 2.3. A net $\left\{F_{\sigma}: \sigma \in \Sigma\right\}$ is evenly-outer-semicontinuous at $x$ if for every $V$ in $\gamma$ and for every $y \in Y$ there is a nbd $U_{x}$ of $x$, a nbd $U_{y}$ of $y$ and a $\sigma_{0} \in \Sigma$ such that, for every $\sigma \geq \sigma_{0}$ and for every $z \in U_{x}$ it results:

$$
F_{\sigma}(z) \cap U_{y} \subset V\left[F_{\sigma}(x)\right] \text {. }
$$

Definition 2.4. A net $\left\{F_{\sigma}: \sigma \in \Sigma\right\}$ is evenly-inner-semicontinuous at $x$ if for every $V$ in $\gamma$ and for every $y \in Y$ there is a nbd $U_{x}$ of $x$, a nbd $U_{y}$ of $y$ and a $\sigma_{0} \in \Sigma$ such that, for every $\sigma \geq \sigma_{0}$ and for every $z \in U_{x}$ it results:

$$
F_{\sigma}(x) \cap U_{y} \subset V\left[F_{\sigma}(z)\right] .
$$

Definition 2.5. A net $\left\{F_{\sigma}: \sigma \in \Sigma\right\}$ is evenly-semicontinuous at $x$ if it is evenly-outer and evenly-inner-semicontinuous at $x$.

If the above definitions are verified for every $x \in X$ we will say that the net is evenly-inner-semicontinuous or evenly-outer-semicontinuous or evenly-semicontinuous (in $X$ ).

Remark 2.6. Notice that if we require that the condition $(*)$ is satisfied for every $\sigma \in \Sigma$, our Definition 2.4 restricted on a net $\left\{f_{\sigma}: \sigma \in \Sigma\right\}$ of singlevalued functions is equivalent to the classical notion of even continuity at $x$ [10].

Let $\left\{f_{\sigma}: \sigma \in \Sigma\right\}$ be evenly continuous at $x$. If $V \in \gamma$ and $y \in Y$ there is $V_{1} \in \gamma$ symmetric, open and such that $V_{1} \circ V_{1} \subset V$. By even continuity of $\left\{f_{\sigma}: \sigma \in \Sigma\right\}$, starting from $x, y$ and $V_{1}[y]$, we can find a nbd $U_{x}$ of $x$ and a nbd $U_{y}$ of $y$ such that $f_{\sigma}\left(U_{x}\right) \subset V_{1}[y]$ whenever $f_{\sigma}(x) \in U_{y}$ and whenever $\sigma \in \Sigma$. We can prove that for every $z \in U_{x}$ and $\sigma \in \Sigma$ it results:

$$
\left\{f_{\sigma}(x)\right\} \cap U_{y} \cap V_{1}[y] \subset V\left[f_{\sigma}(z)\right] .
$$

Indeed, if $\left\{f_{\sigma}(x)\right\} \cap U_{y} \cap V_{1}[y]$ is non empty, $f_{\sigma}(x)$ belongs to $U_{y}$ and $f_{\sigma}(z)$ belongs to $V_{1}[y]$ for every $z \in U_{x}$. So we obtain $\left(f_{\sigma}(x), f_{\sigma}(z)\right) \in V$ since $\left(f_{\sigma}(x), y\right) \in V_{1}$ and $\left(f_{\sigma}(z), y\right) \in V_{1}$.

To prove the opposite, let $\left\{f_{\sigma}: \sigma \in \Sigma\right\}$ be evenly-outer-semicontinuous at $x$ with respect to all $\sigma$. If $y \in Y$ and $U_{y}$ is a nbd of $y$, there is $V$ in $\gamma$ with $V[y] \subset U_{y}$ and $V_{1}$ in $\gamma$ such that $V_{1} \circ V_{1} \subset V$. By assumption there is a nbd $U_{x}$ of $x$ and a nbd $U_{y}^{\prime}$ of $y$ such that for every $z \in U_{x}$ it results

$$
\left\{f_{\sigma}(x)\right\} \cap V_{1}[y] \cap U_{y}^{\prime} \subset V_{1}\left[f_{\sigma}(z)\right] .
$$

Now, if for a $\sigma f_{\sigma}(x) \in V_{1}[y] \cap U_{y}^{\prime}$, it results $f_{\sigma}(x) \in V_{1}\left[f_{\sigma}(z)\right]$, therefore $\left(f_{\sigma}(x), y\right)$ belongs to $V_{1}$ and $f_{\sigma}(z) \in V[y] \subset U_{y}$ for every $z \in U_{x}$.

In the literature we can find notions stronger than even-continuity. Due to Kowalczyk [11], there are the following notions concerning a family $\mathcal{F}$ of set-valued maps with values in a uniform space $(Y, \gamma)$. 
Definition 2.7. $\mathcal{F}$ is upper equicontinuous at $x \in X$ if for every $V \in \gamma$

$(* *)$ there exists a nbd $U_{x}$ of $x$ such that whenever $F \in \mathcal{F}$ and $z \in U_{x}$ then $F(z) \subset V[F(x)]$.

Definition 2.8. $\mathcal{F}$ is lower equicontinuous at $x \in X$ if for every $V \in \gamma$ there exists a nbd $U_{x}$ of $x$ such that whenever $F \in \mathcal{F}$ and $z \in U_{x}$ then $F(x) \subset V[F(z)]$.

Definition 2.9. $\mathcal{F}$ is equicontinuous at $x \in X$ if it is lower and upper equicontinuous at $x$.

All definitions given in 2.7, 2.8 and 2.9 applied to a family $\mathcal{F}$ of singlevalued functions coincide with the classical notion of equicontinuity. It is easy to prove that if a net of set-valued maps is upper-equicontinuous then it is also even-outer-semicontinuous. We will say that a net $\left\{F_{\sigma}: \sigma \in\right.$ $\Sigma\}$ is asymptotically upper equicontinuous if the condition $(* *)$ is satisfied for all $\sigma$ sufficiently large. This notion can be found for example in [9] under the name quasi equi-semicontinuity. If $Y$ is compact then this notion coincides with the notion of even-outer-semicontinuity. In general, however this notion turns out to be too constringent for applications purposes [4].

We have the following characterization of compact spaces.

Theorem 2.10. If $X$ is a non discrete topological space and $Y$ is a Tychonoff space, the following conditions are equivalent:

a) $Y$ is compact;

b) every net of set-valued maps from $X$ to $Y$ is asymptotically upper equicontinuous iff it is evenly-outer-semicontinuous.

Proof. If $Y$ is compact, it is easy to verify that both of the definitions coincide. Let us prove the converse. Suppose $Y$ is not compact, then there is a net $\left\{y_{\sigma}: \sigma \in \Sigma\right.$ \} of points of $Y$ having no cluster point and a point $x_{0}$ in $X$ which is not isolated, therefore in every nbd $U$ of $x_{0}$ we can choose a point $x_{U} \in U \backslash\left\{x_{0}\right\}$. Without loss of generality we can suppose that there is a point $y \in Y$ different from all $y_{\sigma}$. Consider the set $\mathcal{U}\left(x_{0}\right) \times \Sigma$ with the natural order (that is $(U, \sigma) \geq\left(U^{\prime}, \sigma^{\prime}\right)$ iff $U \subset U^{\prime}$ and $\left.\sigma \geq \sigma^{\prime}\right)$. The net $\left\{F_{(U, \sigma)}:(U, \sigma) \in\left(\mathcal{U}\left(x_{0}\right) \times \Sigma\right)\right\}$ defined by

$$
F_{(U, \sigma)}(x)=\left\{\begin{array}{lll}
\{y\} & \text { if } & x \neq x_{U} \\
\left\{y, y_{\sigma}\right\} & \text { if } & x=x_{U}
\end{array}\right.
$$

is not upper equicontinuous at $x_{0}$ with respect to any compatible uniformity $\gamma$ on $Y$. Indeed, if $V \in \gamma$ there is $\sigma_{0}$ in $\Sigma$ such that $y_{\sigma} \notin V[y]$ for all $\sigma \geq \sigma_{0}$; 
thus $F_{(U, \sigma)}\left(x_{U}\right) \not \subset V\left[F_{(U, \sigma)}\left(x_{0}\right)\right]$ for every nbd $U$ of $x_{0}$ and every $\sigma \geq \sigma_{0}$. We can prove that this net is evenly-outer-semicontinuous. Indeed, if $V \in \gamma$, $z \in Y$ and $U_{z}$ is a nbd of $z$ which does not contain $y_{\sigma}$ for all $\sigma \geq \sigma_{0}$, we have

$$
U_{z} \cap F_{(U, \sigma)}(x) \subset V\left[F_{(U, \sigma)}\left(x_{0}\right)\right]
$$

for all $x \in X$ and for all $\sigma \geq \sigma_{0}$.

Due to [4] are the following definitions concerning set-valued maps from a topological space $X$ to a metric space $Y$. If $\mathcal{I}$ is an index space, $\mathcal{H}$ a filter on $\mathcal{I}$ and $\left\{F_{i}: i \in \mathcal{I}\right\}$ a collection of set-valued maps it states:

Definition 2.11. $\left\{F_{i}: i \in \mathcal{I}\right\}$ is equi-outer-semicontinuous at $x_{0}$ if for every compact set $B \subset Y$ and every $\varepsilon \geq 0$ there exists a nbd $V$ of $x_{0}$ and $H \in \mathcal{H}$ such that for every $x \in V$ and every $i \in H$

$$
F_{i}(x) \cap B \subset \varepsilon F_{i}\left(x_{0}\right) .
$$

Definition 2.12. $\left\{F_{i}: i \in \mathcal{I}\right\}$ is equi-inner-semicontinuous at $x_{0}$ if for every compact set $B \subset Y$ and every $\varepsilon \geq 0$ there exists a nbd $V$ of $x_{0}$ and $H \in \mathcal{H}$ such that for every $x \in V$ and every $i \in H$

$$
F_{i}\left(x_{0}\right) \cap B \subset \varepsilon F_{i}(x) .
$$

Definition 2.13. $\left\{F_{i}: i \in \mathcal{I}\right\}$ is equi-semicontinuous at $x_{0}$ if it is both equi-upper and equi-inner semicontinuous at $x_{0}$.

Obviously if the above conditions are verified for every $x \in X,\left\{F_{i}\right.$ : $i \in \mathcal{I}\}$ will be said equi-outer or equi-inner or equi-semicontinuous. Of course we can naturally extend the above Definitions 2.11, 2.12, 2.13 for any net with values in uniform spaces. It is easy to verify that if a net of set-valued maps is evenly-outer (inner)-semicontinuous then it is also equiouter (inner)-semicontinuous and if $Y$ is locally compact then these notions coincide. We can even characterize local compactness by this coincidence as the following theorem states.

Theorem 2.14. If $X$ is a non discrete topological space and $Y$ is a Tychonoff space, the following conditions are equivalent:

a) $Y$ is locally compact;

b) every net of set-valued maps from $X$ to $Y$ is equi-outer-semicontinuous iff it is evenly-outer-semicontinuous. 
Proof. If $Y$ is locally compact, it is easy to verify that condition b) is verified. Now, suppose $Y$ is not locally compact. There is $y \in Y$ which has no compact nbd. Thus, for every open nbd $U$ of $y$ and every $K \in K(Y)$, there is $y_{U, K} \in U \backslash K$. Let $x \in X$ be a non-isolated point and $z \in Y$ be a point different from $y$. For every open nbd $W$ of $x$ choose $x_{W} \in W \backslash\{x\}$.

If we consider $\mathcal{L}=\mathcal{U}(x) \times \mathcal{U}(y) \times K(Y)$ with the natural direction (i.e. $(W, U, K) \leq\left(W^{\prime}, U^{\prime}, K^{\prime}\right)$ iff $\left.W^{\prime} \subset W, U^{\prime} \subset U, K \subset K^{\prime}\right)$ and for every $(W, U, K)$ we put

$$
F_{(W, U, K)}(t)= \begin{cases}\{z\} & \text { if } t \neq x_{W} \\ \left\{z, y_{U, K}\right\} & \text { if } t=x_{W}\end{cases}
$$

we can prove that the net $\left\{F_{(W, U, K)}:(W, U, K) \in \mathcal{L}\right\}$ is equi-outer-semicontinuous at every point with respect to any compatible uniformity $\gamma$. It is sufficient to verify this only at the point $x$. Let $V \in \gamma$ and $K \in K(Y)$. Then for every $v \in X$ and $(W, U, C) \geq(X, Y, K)$ we have

$$
F_{(W, U, C)}(v) \cap K \subset V\left[F_{(W, U, C)}(x)\right] .
$$

However $\left\{F_{(W, U, K)}:(W, U, K) \in \mathcal{L}\right\}$ is not evenly-outer-semicontinuous at $x$. Let $G \in \gamma$ be such that $G[z] \cap G[y]=\emptyset$ and $G$ is open and symmetric. Now for every nbd $W$ of $x$, every nbd $U$ of $y$ and every $(C, D, K) \in \mathcal{L}$ we have

$$
F_{(W \cap C, U \cap D \cap G[y], K)}\left(x_{W \cap C}\right) \cap U=\left\{z, y_{U \cap D \cap G[y], K}\right\} \cap U \not \subset G[z] .
$$

If $X$ and $Y$ are first countable topological spaces then a sequence of set-valued maps is equi-outer-semicontinuous iff it is evenly-outer semicontinuous.

Theorem 2.15. Let $X$ and $Y$ be first countable topological spaces and $(Y, \gamma)$ be a uniform space. A sequence $\left\{F_{n}: n \in \mathbb{Z}^{+}\right\}$of set-valued maps from $X$ to $Y$, is equi-outer-semicontinuous iff it is evenly-outer-semicontinuous.

Proof. Of course it is sufficient to prove that if $\left\{F_{n}: n \in \mathbb{Z}^{+}\right\}$is equiouter-semicontinuous at $x$ then it is also even-outer-semicontinuous at $x$. Suppose that this is not true. This means that there is $V \in \gamma$ and $y \in Y$ such that for every nbd $W$ of $x, U$ of $y$ and for every $m \in \mathbb{Z}^{+}$there exists $n(W, U, m) \geq m$ and a point $x_{n(W, U, m)}$ in $W$ such that

$$
F_{n(W, U, m)}\left(x_{n(W, U, m)}\right) \cap U \not \subset V\left[F_{n(W, U, m)}(x)\right] .
$$

Let $\left\{W_{n}\right\}$ and $\left\{U_{n}\right\}$ be a countable base of nbds of $x$ and $y$ respectively. Thus for every $n \in \mathbb{Z}^{+}$there is $m_{n} \geq n, x_{n}$ and $y_{n}$ such that $x_{n} \in W_{n}$, $y_{n} \in F_{m_{n}}\left(x_{n}\right) \cap U_{n} \backslash V\left[F_{m_{n}}\left(x_{n}\right)\right]$. Of course $\left\{x_{n}: n \in \mathbb{Z}^{+}\right\}$converges to $x$ 
and $\left\{y_{n}: n \in \mathbb{Z}^{+}\right\}$converges to $y$. Consider the compact set $B=\left\{y_{n}\right.$ : $\left.n \in \mathbb{Z}^{+}\right\} \cup\{y\}$. By assumption there must exist a nbd $W^{\prime}$ of $x$ and $n_{0} \in \mathbb{Z}^{+}$ with

$$
F_{n}(r) \cap B \subset V\left[F_{n}(x)\right]
$$

for every $n \geq n_{0}$ and for every $r \in W^{\prime}$, but this is a contradiction since, if $n \geq n_{0}$ is such that $W_{n} \subset W^{\prime}$, we have

$$
y_{n} \in F_{m_{n}}\left(x_{n}\right) \cap B \backslash V\left[F_{m_{n}}(x)\right] .
$$

\section{Pointwise and graph convergence}

In this part we prove the main result of our paper. We show that evenouter-semicontinuity allows us to pass from the pointwise convergence to the graph convergence and vice versa. We also prove that if a net is simultaneously pointwise and graph convergent then it has to be evenly-outersemicontinuous.

Theorem 3.1. Let $X$ be a topological space and $(Y, \gamma)$ be a uniform space. Suppose that $\left\{F_{\sigma}: \sigma \in \Sigma\right\}$ is evenly-outer-semicontinuous and pointwise convergent to $F$. Then it is also graph convergent to $F$.

Proof. Of course Gr $F \subset \operatorname{Li~Gr} F_{\sigma}$. To prove that Ls Gr $F_{\sigma} \subset \operatorname{Gr} F$, we will show that if $(x, y) \in \operatorname{Ls} \operatorname{Gr} F_{\sigma}$ then $y \in \operatorname{Ls} F_{\sigma}(x)$. If $U_{y}$ is a nbd of $y$ and $\sigma$ an element of $\Sigma$, we will find $\eta \geq \sigma$ such that $F_{\eta}(x) \cap U_{y} \neq \emptyset$. There is $V_{1}$ (open and symmetric) in $\gamma$ satisfying $V_{1}[y] \subset U_{y}$ and $V_{2}$ (open and symmetric) in $\gamma$ satisfying $V_{2} \circ V_{2} \subset V_{1}$. Since $\left\{F_{\sigma}: \sigma \in \Sigma\right\}$ is evenlyouter-semicontinuous there is a nbd $U_{x}$ of $x$, a nbd $U_{y}^{\prime}$ of $y$ and $\sigma_{0} \in \Sigma$ with

$$
F_{\sigma}(z) \cap U_{y}^{\prime} \subset V_{2}\left[F_{\sigma}(x)\right]
$$

for every $z \in U_{x}$ and every $\sigma \geq \sigma_{0}$. Without loss of generality we can suppose that $U_{y}^{\prime} \subset V_{2}[y]$. Since $U_{x} \times U_{y}^{\prime}$ is a nbd of $(x, y) \in \operatorname{Ls} \operatorname{Gr} F_{\sigma}$, there is $\eta \geq \sigma, \eta \geq \sigma_{0}$ with

$$
\operatorname{Gr} F_{\eta} \cap\left(U_{x} \times U_{y}^{\prime}\right) \neq \emptyset \text {. }
$$

If we choose $\left(z_{\eta}, y_{\eta}\right) \in \operatorname{Gr} F_{\eta} \cap\left(U_{x} \times U_{y}^{\prime}\right)$, from $(\square)$ we obtain

$$
y_{\eta} \in F_{\eta}\left(z_{\eta}\right) \cap U_{y}^{\prime} \subset V_{2}\left[F_{\eta}(x)\right] .
$$

Thus there is $v_{\eta} \in F_{\eta}(x)$ with $\left(y_{\eta}, v_{\eta}\right) \in V_{2}$, and, being $\left(y_{\eta}, y\right) \in V_{2}$, it results that

i.e. $V_{1}[y] \cap F_{\eta}(x) \neq \emptyset$.

$$
\left(v_{\eta}, y\right) \in V_{2} \circ V_{2} \subset V_{1},
$$


Theorem 3.2. Let $X$ be a topological space and $(Y, \gamma)$ be a uniform one. Suppose that $\left\{F_{\sigma}: \sigma \in \Sigma\right\}$ is evenly-outer-semicontinuous and graph convergent to $F$. Then it is also pointwise convergent to $F$.

Proof. Of course Ls $F_{\sigma}(x) \subset F(x)$. Now let $y \in F(x)$ and let $U_{y}$ be a nbd of $y$. We will show that there is $\sigma_{0} \in \Sigma$ for which

$$
F_{\sigma}(x) \cap U_{y} \neq \emptyset
$$

for every $\sigma \geq \sigma_{0}$. There are $V_{1}$ and $V_{2}$ (both open and symmetric) in $\gamma$ satisfying $V_{1}[y] \subset U_{y}$ and $V_{2} \circ V_{2} \subset V_{1}$. Since $\left\{F_{\sigma}: \sigma \in \Sigma\right\}$ is evenlyouter-semicontinuous, there is a nbd $U_{x}$ of $x$, a nbd $U_{y}^{\prime}$ of $y$ and $\sigma_{0} \in \Sigma$ with

$$
F_{\sigma}(z) \cap U_{y}^{\prime} \subset V_{2}\left[F_{\sigma}(x)\right]
$$

for every $z \in U_{x}$ and every $\sigma \geq \sigma_{0}$. Without loss of generality we can suppose that $U_{y}^{\prime} \subset V_{2}[y]$. Since $U_{x} \times U_{y}^{\prime}$ is a nbd of $(x, y) \in \operatorname{Li} \operatorname{Gr} F_{\sigma}$, there is $\sigma_{1} \in \Sigma$ such that for every $\sigma \geq \sigma_{1}$

$$
\text { Gr } F_{\sigma} \cap\left(U_{x} \times U_{y}^{\prime}\right) \neq \emptyset \text {. }
$$

For $\sigma \geq \sigma_{0}$ and $\sigma \geq \sigma_{1}$, choose

$$
\left(x_{\sigma}, y_{\sigma}\right) \in \operatorname{Gr} F_{\sigma} \cap\left(U_{x} \times U_{y}^{\prime}\right) .
$$

We obtain that $\left(y_{\sigma}, y\right) \in V_{2}$ and

$$
y_{\sigma} \in F_{\sigma}\left(x_{\sigma}\right) \cap U_{y}^{\prime} \subset V_{2}\left[F_{\sigma}(x)\right] .
$$

We can conclude that there is $v_{\sigma} \in F_{\sigma}(x)$ satisfying $\left(y_{\sigma}, v_{\sigma}\right) \in V_{2}$ that is $v_{\sigma} \in V_{1}[y] \subset U_{y}$.

Theorem 3.3. Let $X$ be a topological space and $(Y, \gamma)$ be a uniform one. Suppose that $\left\{F_{\sigma}: \sigma \in \Sigma\right\}$ graph and pointwise converges to $F$. Then it is evenly-outer-semicontinuous.

Proof. Suppose this is not true for a point $x \in X$. There is $V$ in $\gamma$ and $y \in Y$ such that for every nbd $U$ of $x$, every nbd $O$ of $y$ and every $\sigma \in \Sigma$, there is $\eta(U, O, \sigma) \in \Sigma, \eta(U, O, \sigma) \geq \sigma$ and $x_{\eta(U, O, \sigma)} \in U$ for which

$$
F_{\eta(U, O, \sigma)}\left(x_{\eta(U, O, \sigma)}\right) \cap O \not \subset V\left[F_{\eta(U, O, \sigma)}(x)\right] .
$$

This allows us to choose a net

$$
y_{\eta(U, O, \sigma)} \in F_{\eta(U, O, \sigma)}\left(x_{\eta(U, O, \sigma)}\right) \cap O \backslash V\left[F_{\eta(U, O, \sigma)}(x)\right]
$$

converging to $y$. It is easy to verify that

$$
\mathcal{L}=\{\eta(U, O, \sigma): U \in \mathcal{U}(x), O \in \mathcal{U}(y), \sigma \in \Sigma\}
$$


is a cofinal family in $\Sigma$, thus

$$
\left\{F_{\eta(U, O, \sigma)}: U \in \mathcal{U}(x), O \in \mathcal{U}(y), \sigma \in \Sigma\right\}
$$

is a subnet of $\left\{F_{\sigma}: \sigma \in \Sigma\right\}$.

We will show that $(x, y) \in \operatorname{Ls} \operatorname{Gr} F_{\eta(U, O, \sigma)}$. Let $G \in \mathcal{U}(x), H \in \mathcal{U}(y)$ and $\eta(U, O, \sigma) \in \mathcal{L}$. By the assumption we can find $\eta(G, H, \eta(U, O, \sigma)) \geq$ $\eta(U, O, \sigma)$ and the related points satisfying the conditions:

$$
x_{\eta(G, H, \eta(U, O, \sigma))} \in G
$$

and

$$
y_{\eta(G, H, \eta(U, O, \sigma))} \in H \cap F_{\eta(G, H, \eta(U, O, \sigma))}\left(x_{\eta(G, H, \eta(U, O, \sigma))}\right) .
$$

Since $(x, y) \in \operatorname{Ls} F_{\eta(U, O, \sigma)} \subset \operatorname{Ls} F_{\sigma}$, we have $y \in F(x)$. There is $V_{1} \in \gamma$ such that $V_{1}$ is open, symmetric and $V_{1} \circ V_{1} \subset V$. Being $F(x)=\operatorname{Li} F_{\sigma}(x)$, there is $\sigma_{0} \in \Sigma$ with $F_{\sigma}(x) \cap V_{1}[y] \neq \emptyset$ for every $\sigma \geq \sigma_{0}$. Then for $\eta\left(X, V_{1}[y], \sigma_{0}\right)$ we have

$$
F_{\eta\left(X, V_{1}[y], \sigma_{0}\right)}(x) \cap V_{1}[y] \neq \emptyset
$$

and

$y_{\eta\left(X, V_{1}[y], \sigma_{0}\right)} \in F_{\eta\left(X, V_{1}[y], \sigma_{0}\right)}\left(x_{\eta\left(X, V_{1}[y], \sigma_{0}\right)}\right) \cap V_{1}[y] \subset\left(V_{1} \circ V_{1}\right)\left[F_{\eta\left(X, V_{1}[y], \sigma_{0}\right)}(x)\right]$ which is in contradiction with $(\triangle)$.

For nets of single-valued functions we obtain the following result $([5,12$, $13])$ :

Corollary 3.4. Let $X$ be a topological space and $Y$ be a uniform one. Let $f$ be a single-valued function from $X$ to $Y$ and $\left\{f_{\sigma}: \sigma \in \Sigma\right\}$ be a net of single-valued functions from $X$ to $Y$. Consider the following conditions:

a) $\left\{f_{\sigma}: \sigma \in \Sigma\right\}$ converges pointwise to $f$;

b) $\left\{f_{\sigma}: \sigma \in \Sigma\right\}$ graph converges to $f$;

c) $\left\{f_{\sigma}: \sigma \in \Sigma\right\}$ is evenly-outer-semicontinuous.

Then any two of these conditions imply the other.

The following example shows that even if we consider a sequence of continuous single-valued functions the condition c) in the above corollary cannot be replaced by

c') $\left\{f_{\sigma}: \sigma \in \Sigma\right\}$ is evenly-continuous.

Let $X=\{0\} \cup\left\{1 / n ; n \in \mathbb{Z}^{+}\right\}$with the usual topology and, for every $n$, put

$$
f_{n}(x)=\left\{\begin{array}{lll}
0 & \text { if } & x \neq 1 / n \\
n & \text { if } & x=1 / n
\end{array}\right.
$$


Then $\left\{f_{n}\right\}$ is a sequence of continuous functions pointwise and graph convergent to $f$, where $f(x)=0$ at every $x \in X$. Of course $\left\{f_{n}\right\}$ is not evenly continuous.

Let us observe also that for sequences of continuous single-valued functions the notions of even continuity and even-outer-semicontinuity are independent. It is easy to prove that the sequence $([13])$

$$
f_{n}(x)=\left\{\begin{array}{lll}
0 & \text { if } & 1 / n \leq x \leq 1 \\
n-x n^{2} & \text { if } & 0 \leq x<1 / n
\end{array}\right.
$$

is evenly continuous but not evenly-outer-semicontinuous.

\section{Continuous convergence}

Definition 4.1. Let $X$ and $Y$ be topological spaces. A net $\left\{F_{\sigma}: \sigma \in \Sigma\right\}$ of set-valued maps from $X$ to $Y$ converges continuously to a set-valued map $F$ if for every $x \in X$ and every net $\left\{x_{\alpha}: \alpha \in A\right\}$ convergent to $x$, the net $\left\{F_{\sigma}\left(x_{\alpha}\right):(\sigma, \alpha) \in \Sigma \times A\right\}$ Painlevé-Kuratowski converges to $F(x)$.

Obviuosly continuous convergence implies pointwise and graph convergence (and therefore even-outer-semicontinuity).

The following proposition shows that this convergence implies as well even-inner-semicontinuity.

Proposition 4.2. If a net $\left\{F_{\sigma}: \sigma \in \Sigma\right\}$ continuously converges to $F$, then $\left\{F_{\sigma}: \sigma \in \Sigma\right\}$ is evenly-semicontinuous.

Proof. It is sufficient to prove that the net $\left\{F_{\sigma}: \sigma \in \Sigma\right\}$ is evenly-innersemicontinuous. If it is not true at a point $x \in X$, then there exists $V$ in $\gamma$ and $y \in Y$ such that for every nbd $U$ of $x$, every nbd $O$ of $y$ and every $\sigma \in \Sigma$ there is $\eta(U, O, \sigma) \in \Sigma, \eta(U, O, \sigma) \geq \sigma, x_{(U, O, \sigma)} \in U$ for which

$$
F_{\eta(U, O, \sigma)}(x) \cap O \not \subset V\left[F_{\eta(U, O, \sigma)}\left(x_{(U, O, \sigma)}\right)\right] .
$$

This allows us to choose a net

$$
y_{\eta(U, O, \sigma)} \in F_{\eta(U, O, \sigma)}(x) \cap O \backslash V\left[F_{\eta(U, O, \sigma)}\left(x_{\eta(U, O, \sigma)}\right)\right]
$$

converging to $y$. It is easy to verify that

$$
\mathcal{L}=\{\eta(U, O, \sigma): U \in \mathcal{U}(x), O \in \mathcal{U}(y), \sigma \in \Sigma\}
$$

is a cofinal family in $\Sigma$, thus

$$
\left\{F_{\eta(U, O, \sigma)}: U \in \mathcal{U}(x), O \in \mathcal{U}(y), \sigma \in \Sigma\right\}
$$

is a subnet of $\left\{F_{\sigma}: \sigma \in \Sigma\right\}$. 
Since $(x, y) \in \operatorname{Ls} F_{\eta(U, O, \sigma)}$ we obtain $y \in F(x)$. Let $V_{1}$ be a symmetric open element from $\gamma$ with $V_{1} \circ V_{1} \subset V$. The net

$$
\left\{x_{(U, O, \sigma)}:(U, O, \sigma) \in \mathcal{U}(x) \times \mathcal{U}(y) \times \Sigma\right\}
$$

converges to $x$ (considering the natural direction on $\mathcal{U}(x) \times \mathcal{U}(y) \times \Sigma$ ) thus

$$
\left\{F_{\sigma}\left(x_{(U, O, \sigma)}\right): \sigma \in \Sigma,(U, O, \sigma) \in \mathcal{U}(x) \times \mathcal{U}(y) \times \Sigma\right\}
$$

Painlevé-Kuratowski converges to $F(x)$ by assumption. Thus we can find $\sigma_{0} \in \Sigma$ and $(U, O, \sigma) \in \mathcal{U}(x) \times \mathcal{U}(y) \times \Sigma$; such that for every $\sigma \geq \sigma_{0}$ and every $\left(U^{\prime}, O^{\prime}, \sigma^{\prime}\right) \geq(U, O, \sigma)$

$$
F_{\sigma}\left(x_{\left(U^{\prime}, O^{\prime}, \sigma^{\prime}\right)}\right) \cap V_{1}[y] \neq \emptyset .
$$

Let $\sigma^{\prime} \in \Sigma$ be such that $\sigma^{\prime} \geq \sigma$ and $\sigma^{\prime} \geq \sigma_{0}$. If we put $\eta^{\prime}=\eta(U, O \cap$ $\left.V_{1}[y], \sigma^{\prime}\right)$, it results that $\eta^{\prime} \in \mathcal{L}$ and

$$
y_{\eta^{\prime}} \in V_{1}[y] \subset V_{1} \circ V_{1}\left[F_{\eta^{\prime}}\left(x_{\left(U, O \cap V_{1}[y], \sigma^{\prime}\right)}\right)\right] \subset V\left[F_{\eta^{\prime}}\left(x_{\left(U, O \cap V_{1}[y], \sigma^{\prime}\right)}\right)\right]
$$

and this is a contradiction.

Since the continuous convergence at $x$ implies that $\left\{F_{\sigma}: \sigma \in \Sigma\right\}$ is evenly-outer-semicontinuous at $x$, the net has to be evenly-semicontinuous.

Proposition 4.3. If a net $\left\{F_{\sigma}: \sigma \in \Sigma\right\}$ graph converges to $F$ and is enenlysemicontinuous then $\left\{F_{\sigma}: \sigma \in \Sigma\right\}$ converges continuosly to $F$.

Proof. Let $\left\{x_{\alpha}: \alpha \in A\right\}$ be a net in $X$ convergent to $x$. It is sufficient to prove that $F(x) \subset \operatorname{Li} F_{\sigma}\left(x_{\alpha}\right)$. Let $y \in F(x)$ and $O$ be a nbd of $y$ (we will find $\sigma_{0} \in \Sigma$ and $\alpha_{0} \in A$ such that for every $\sigma \geq \sigma_{0}$ and $\alpha \geq \alpha_{0}$ it results $\left.F_{\sigma}\left(x_{\alpha}\right) \cap O \neq \emptyset\right)$.

Let $U \in \gamma$ be such that $U[y] \subset O$, let further $V_{1}$ be an open symmetric element from $\gamma$ with $V_{1} \circ V_{1} \subset U$. Since $\left\{F_{\sigma}: \sigma \in \Sigma\right\}$ is evenly-innersemicontinuous at $x$, there exist a nbd $W$ of $y$ satisfying $W \subset V_{1}[y]$, a nbd $I$ of $x$, an index $\sigma_{1} \in \Sigma$ such that

$$
F_{\sigma}(x) \cap W \subset V_{1}\left[F_{\sigma}(z)\right]
$$

for every $z \in I$ and every $\sigma \geq \sigma_{1}$.

Since $F(x)=\operatorname{Li} F_{\sigma}(x)$, there is $\sigma_{2} \in \Sigma$ such that for every $\sigma \geq \sigma_{2}$

$$
F_{\sigma}(x) \cap W \neq \emptyset \text {. }
$$

There is $\alpha_{0} \in A$ such that $x_{\alpha} \in I$ for every $\alpha \geq \alpha_{0}$. Let $\sigma_{0} \in \Sigma$ be such that $\sigma_{0} \geq \sigma_{1}$ and $\sigma_{0} \geq \sigma_{2}$. We claim that for every $\alpha \geq \alpha_{0}$ and $\sigma \geq \sigma_{0}$

$$
F_{\sigma}\left(x_{\alpha}\right) \cap O \neq \emptyset .
$$

Indeed if $\alpha \geq \alpha_{0}$ and $\sigma \geq \sigma_{0}$ take $y_{\sigma} \in F_{\sigma}(x) \cap W$, by using condition $(\diamond)$ it results $y_{\sigma} \in V_{1}\left[F_{\sigma}\left(x_{\alpha}\right)\right]$. Let $z_{\sigma, \alpha} \in F_{\sigma}\left(x_{\alpha}\right)$ with $\left(y_{\sigma}, z_{\sigma, \alpha}\right) \in V_{1}$. Since 
$y_{\sigma} \in V_{1}[y]$ we have $\left(z_{\sigma, \alpha}, y\right) \in V_{1} \circ V_{1}$ that is $z_{\sigma, \alpha} \subset U[y] \subset O$. Thus $z_{\sigma, \alpha} \in F_{\sigma}\left(x_{\alpha}\right) \cap O$.

\section{References}

[1] Attouch, H., Variational Convergence for Functions and Operators, Appl. Math. Ser., Pitman, London, 1984.

[2] Aubin, J. P., Frankowska, H., Set-valued Analysis, Birhauser, Cambridge, MA, 1990.

[3] Back, K., Concepts of similarity for utility functions, J. Math. Econom. 15 (1986), 129-142.

[4] Bagh, A., Wets, R. J. B., Convergence of set valued mappings: equi-outersemicontinuity, Set-Valued Anal. 4 (1996), 333-360.

[5] Beer, G., More on convergence of continuous functions and topological convergence of sets, Canad. Math. Bull. 28(1) (1985).

[6] Beer, G., Topologies on Closed and Closed Convex Sets, Kluwer Acad. Publ., Dordrecht, 1993.

[7] Beer, G., Lucchetti, R., Convergence of epigraphs and of sublevel sets, Set-Valued Anal. 1 (1993), 159-183.

[8] Brandi, P., Ceppitelli, R., Holá, Ц̆., Topological properties of a new graph topology, J. Convex Anal., to appear.

[9] Dolecki, S., Tangency and differentiation: some applications of convergence theory, Ann. Mat. Pura Appl. 130 (1982), 223-255).

[10] Kelley, J. L., General Topology, Princeton, New York, 1955.

[11] Kowalczyk, S., Topological convergence of multivalued maps and topological convergence of graphs, Demonstratio Math. 27(1) (1994), 79-87.

[12] Naimpally, S., Topological convergence and uniform convergence, Czechoslovak Math. J. 37(112) (1987), 608-612.

[13] Poppe, H., Convergence of evenly continuous nets in general function spaces, Real Anal. Exchange 18(2) (1992-1993), 459-464.

[14] Rockafellar, R. T., Wets, R. J. B., Variational Analysis, Springer, Berlin, 1998.

I. Del Prete

Dipartimento Di Matematica

E APPLICAZIONI

Università DEGLI StUdi di NAPOLI

Via Claudio 21, 80125 Napoli

ITALY

E-MAIL:DELPRETE@CDS.UNINA.IT

$\breve{L}$. HOLÁ

ACADEMY OF SCIENCES

INSTITUT OF MATHEMATICS

S̆TEFÁNIKOVA 49

81473 Bratislava, Slovakia

E-MAIL: HOLA@MAU.SAVBA.SK
M. Di IORIO

Dipartimento Di Matematica

E Applicazioni

Università DEGLI STUd DI NAPOLI

Via Claudio 21, 80125 NAPOLI

ITALY

E-MAIL:DIIORIO@CDS.UNINA.IT 